「材料」(Journal of the Society of Materials Science, Japan), Vol. 67, No. 7, pp. 746-753, July 2018

Original Papers

\title{
Use of Collagen Sheets Simulating Various Skin Types and Age Groups to Evaluate Human Skin Damage Caused by Ultraviolet Light
}

\author{
by \\ Tetsuya TAKAHASHI*, Takayuki OGURA ${ }^{* *}$, Keisuke TANAKA ${ }^{* *}$, Shunji HATTORI**, \\ and Katsumi YOSHINO***
}

\begin{abstract}
Collagen sheets with melanin added or Maillard cross-linking were prepared as models to simulate various human skin types and age groups. The treated collagen sheets were used to study effects of ultraviolet light on the human skin. Extracts were obtained from collagen sheets after ultraviolet light irradiation and analyzed. The results revealed that ultraviolet light irradiation of up to 25 hours mainly formed cross-linkages in collagen sheets with melanin added at $10 \%$ or lower, irradiation for 200 hours mainly cleaved collagen molecule chains. In contrast, ultraviolet light was found to promote cross-linkage formation in collagen molecule chains in the collagen sheet with melanin added at $50 \%$, but hardly cleaved the molecule chains even for long durations of ultraviolet light irradiation. Moreover, ultraviolet light irradiation did not cleave the molecule chains but maintained cross-linkage in collagen sheets that were cross-linked by Maillard processes with glyoxal solutions of $0.1 \mathrm{mM}$ or higher. However, exposure to ultraviolet light irradiation for as long as 200 hours cleaved collagen molecule chains. These results demonstrate that collagen sheets that simulate various human skin types could be useful for studying human skin damage caused by ultraviolet light.
\end{abstract}

Key words:

Ultraviolet Light, Collagen, Skin types, age groups, Melanin, Maillard cross-linking

\section{Introduction}

The Meteorological Agency has used the UV index to provide information on ultraviolet light since 2005 so that the public can take effective measures against ultraviolet light. The World Health Organization and the Ministry of Environment also recommend measures against ultraviolet light ${ }^{1), 2)}$. Wrinkles, sags, and blemishes may be cited as effects of ultraviolet light on the human $\operatorname{skin}^{3), 4)}$. However, the same daily dose of ultraviolet light may manifest largely different skin damage depending on skin type. According to an international classification of skin types, there are six types from Type I for Caucasians to Type VI for Africans. Types II through IV are assigned to Mongoloids. The effects of ultraviolet light depend not only on skin type ${ }^{5), 6)}$ but also on age. Collagen molecules are cross-linked to decrease the elasticity of the skin over time ${ }^{7}$. In addition, cell division is more active in growing children than in adults. Exposure to ultraviolet light in childhood is believed to manifest adverse effects more frequently even if children and adults are exposed to the same daily dose of ultraviolet light.

As explained above, human skin damage caused by ultraviolet light varies by skin type and age. The authors previously used a collagen sheet as a model for human skin while studying the effects of ultraviolet light on the skin ${ }^{8), 9)}$. In this study, we improved the collagen sheet and prepared different versions: some contained melanin added to simulate new and different skin types; other sheets were Maillard-crosslinked in order to approximate skin belonging to different age groups. These collagen sheets were used as models to study human skin damage caused by ultraviolet light.

\section{Experiments}

\subsection{Materials}

\subsubsection{Collagen sheets simulating different skin types}

A solution of pepsin-solubilized collagen (Nippi. Inc.) from porcine dermis was neutralized with a phosphate-buffered physiological saline and heated to $37{ }^{\circ} \mathrm{C}$ for the reconstitution of collagen fibers. After gently stirring, a prescribed amount of eumelanin (Wako Pure Chemical Industries) was added and stirring continued. The suspension of dispersed fibers was vacuum filtered with a Büchner funnel to deposit collagen fibers on a quantitative filter paper (ADVANTEC) $50 \mathrm{~mm}$ in inner diameter. Collagen fibers deposited on the paper were freeze dried to obtain a collagen sheet. The amount of added melanin was $0 \%, 0.1 \%, 1 \%, 10 \%$, and $50 \%$, to simulate the amount of collagen fibers in various skin types. Fig. 1 (a) shows a schematic image of a collagen sheet with melanin added.

2.1.2 Collagen sheets simulating skins of different age

$+\quad$ Received Aug. 4, 2017 (C) 2018 The Society of Materials Science, Japan

* Member: Faculty of Human Sciences, Shimane University, Nishikawatsu-cho, Matsue, 690-8504, Japan

** Nippi Research Institute of Biomatrix, Kuwahara, Toride, 302-0017, Japan

*** Member: Shimane Institute for Industrial Technology, Hokuryo-cho, Matsue, 690-0816, Japan 
groups A solution of pepsin-solubilized collagen (Nippi, Inc.) from porcine dermis was neutralized with a phosphate-buffered physiological saline and heated to $37{ }^{\circ} \mathrm{C}$ for the reconstitution of collagen fibers. After gently stirring, a glyoxal solution (Kanto Chemical Co., Inc.) was added to the solution at a ratio of $50 \mathrm{~mL}$ to $75 \mathrm{mg}$ of collagen. After stirring, the mixed solution was kept at $37{ }^{\circ} \mathrm{C}$ to react for $24 \mathrm{~h}$. Glyoxal solutions at concentrations of $0 \mathrm{mM}, 0.01 \mathrm{mM}$, $0.1 \mathrm{mM}, 1 \mathrm{mM}$, and $10 \mathrm{mM}$ were used for the reaction. The method described above was used to deposit dispersed collagen fibers on filter paper, with deposits then freeze dried to prepare collagen sheets. Glyoxal is a compound derived from glucose, a sugar, through autoxidaton that possesses carbonyl groups. Glyoxal is highly reactive and induces the Maillard reaction that results in a final, cross-linked Maillard reaction product (Advanced Glycation End Products: AGE). Fig. 1 (b) shows a schematic image of a Maillard cross-linked collagen sheet.

(a)

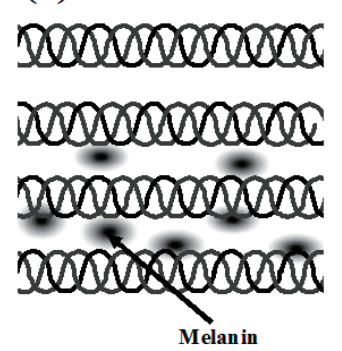

(b)

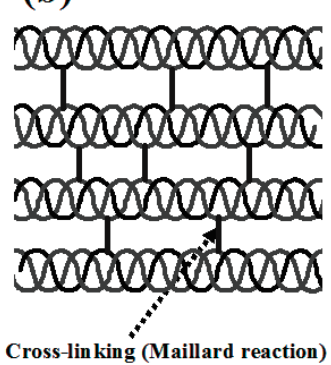

Fig. 1 Schematic images of prepared collagen sheets.

(a) Collagen sheet with melanin added, (b) collagen sheet Maillard cross-linked with glyoxal

\subsection{Methods for ultraviolet light irradiation}

SUNTEST CPS, a Xenon tester produced by Shimadzu Corporation (Kyoto, Japan), was used to irradiate collagen sheets for 0.08-200 hours. Collagen sheets containing added melanin were placed with the surface of denser melanin distribution on the filter paper side up on the stand. A digital UV detector, UVX, from Ultraviolet Products, LLC (Cambridge, UK), was used to measure the irradiation intensity. The following measurements were obtained: 9.260 $\mathrm{mW} / \mathrm{cm}^{2}(365 \mathrm{~nm}), 8.420 \mathrm{~mW} / \mathrm{cm}^{2}(310 \mathrm{~nm})$, and 1.291 $\mathrm{mW} / \mathrm{cm}^{2}(254 \mathrm{~nm})$. The atmospheric temperature in the irradiation chamber was $38.5^{\circ} \mathrm{C}$ during the irradiation.

\subsection{Preparation of extracts from collagen sheets}

The collagen was extracted to analyze the collagen sheets. A sheet of the exposed collagen was fine cut to a size of $3 \times 3$ $\mathrm{mm}$, and approximately $0.1 \mathrm{~g}$ of the cut sheet was transferred to a weighing bottle and immersed in a 500-fold volume of a $50 \mathrm{mM}$ aqueous acetic acid solution. A shaker was used to shake the bottle at a speed of $120 \mathrm{rpm}$ for $24 \mathrm{~h}$. The supernatant was collected to obtain the collagen extract.

\subsection{Measurement methods}

2.4.1 Observation under a scanning electron microscope A carbon tape for vapor deposition was pasted on a stage for electron microscopy, and a sample was fixed onto the tape. After drying the fixed sample at room temperature for $24 \mathrm{~h}$, gold was vapor-deposited on the sample using an ion sputter E-1010 (Hitachi High-Technologies Corporation). Then, an S-3000N scanning electron microscope (SEM), (Hitachi Science Systems Co., Ltd.), was used to observe the surface of the exposed collagen sheets. SEM was performed at an accelerating voltage of $20 \mathrm{kV}$.

2.4.2 Changes in color of exposed collagen sheets To study changes in the color of the exposed collagen sheets, reflectance of the sheets was measured using a UV-3100 spectrophotometer (Shimadzu Corporation), to which the integrating sphere was attached. Spectra for a wavelength range of 220-700 nm were obtained under the conditions of a 2.0-nm slit-width and a $0.5-\mathrm{nm}$ sampling-pitch. Barium sulfate powder was caked to prepare a plate, which was used as a standard white plate. COL-UVPC, a software program for color measurement (Shimadzu Co., Ltd.), was used to measure the luminosity $\left(\mathrm{L}^{*}\right)$ of collagen sheets.

2.4.3 Quantification of protein concentrations of extracts A mixture of $42 \mathrm{~mL}$ of a $1.83 \times 10^{-2} \mathrm{~mol} / \mathrm{L}$ aqueous copper(II) sulfate solution and $80 \mathrm{~mL}$ of a 9.49 $\mathrm{mol} / \mathrm{L}$ aqueous sodium hydroxide solution was stirred to prepare a burette reagent, $500 \mu \mathrm{L}$ of which was added to 1000 $\mu \mathrm{L}$ of the collagen extract. The mixture was stirred and left standing for $10 \mathrm{~min}$. A spectrophotometer was used to measure the absorbance at $310 \mathrm{~nm}$ to calculate the total protein concentration of the collagen extract. In the sample containing melanin, the quantity of melanin added was adjusted so that the amount of collagen become the same, as determined by the protein concentrations. Different concentrations of the collagen standard solutions $(0.01-0.10 \%)$ were used to quantify the total protein.

2.4.4 Quantification of terminal amino group concentration of extracts In a test tube containing 0.1 $\mathrm{mL}$ of a collagen extract, $1 \mathrm{~mL}$ of a ninhydrin solution (propyleneglycol monomethyl ether, ninhydrin; Wako Pure Chemical Industries Co., Ltd.), and $1 \mathrm{~mL}$ of a buffer solution (propyleneglycol monomethyl ether, lithium acetate dehydrate; Wako Pure Chemical Industries Co., Ltd), were added. The mixture was heated at $100{ }^{\circ} \mathrm{C}$ for $10 \mathrm{~min}$ and left standing at room temperature for about $3 \mathrm{~h}$. A spectrophotometer was used to measure sample solutions for absorbance at $570 \mathrm{~nm}$.

A molecule of bovine albumin has one terminal amino group, as do collagen molecule chains. Different 
concentrations of bovine albumin solutions were prepared and treated in the same way as the collagen extracts to measure absorbance. Assuming that ninhydrin reacts only with the terminal amino groups, a working curve was prepared for the terminal amino group concentration and absorbance. Terminal amino group concentrations of the test solutions were calculated from absorbance measures. In the sample containing melanin, the quantity of melanin added was adjusted so that the amount of collagen become the same, as determined by the terminal amino group concentration.

\subsubsection{Sodium dodecyl sulfate-polyacrylamide gel} electrophoresis (SDS-PAGE) A Mini-Protean 3 cell (Bio-Rad Japan Co., Ltd.) was used for electrophoresis. First, a freeze-drier FD-1000 (Tokyo Rikakikai Co., Ltd.), was used to freeze-dry and concentrate $0.1 \mathrm{~mL}$ aliquots of collagen extracts. To a concentrated collagen extract, $15 \mu \mathrm{L}$ of a Laemmli sample buffer containing 5\% 2-mercaptoethanol was added, and the mixture was heated at $95{ }^{\circ} \mathrm{C}$ for $5 \mathrm{~min}^{10)}$. The whole test sample solution was injected into a well of the gel, and electrophoresis was performed at a constant voltage of $100 \mathrm{~V}$ for about $2 \mathrm{~h}$. Then, a $5 \%$ stacking gel $1.0 \mathrm{~mm}$ in thickness and a $15 \%$ separation gel were prepared. In the sample containing melanin, the quantity of melanin was also adjusted to control the amount of the solution injected in to a well of the gel so that the amount of collagen becomes the same.

Bands were stained with Coomassie Brilliant Blue R-250 for detection. As molecular-weight markers, $6 \mu \mathrm{L}$ of Precision Plus Protein ${ }^{\mathrm{TM}}$ All Blue Standards was used.

\section{Results and Discussion}

\subsection{Observation of the fine structure of collagen sheet}

Melanocytes are stimulated by ultraviolet light to produce melanin. The melanin moves toward the skin surface and is oxidized by ultraviolet light resulting in black pigmentation. This melanin is considered an important substance to protect the body because it absorbs ultraviolet light. In addition, Maillard reactions in collagen result in cross-linked structures. This so-called Maillard cross-linking is said to form mainly during aging. Therefore, collagen sheets were prepared with melanin added or Maillard cross-linked with glyoxal as models to simulate various skin types and age groups.

Melanin tends to be deposited more on the filter paper side (down side) in the process of collagen sheet preparation. This resulted in a large difference in luminosity between the front and rear sides of the collagen sheets. The absorbance spectrum was examined by reflected light for the filter paper side (lower side) and the opposite side (upper side) of a collagen sheet with melanin added at 50\%. Measurements were also taken for both sides of a collagen sheet that contained no added melanin and was not cross-linked.
The results showed a large difference in absorbance between the two sides of the collagen sheet with melanin added at $50 \%$. Absorbance ranged from 1.02 to 1.10 (luminosity $\mathrm{L}^{*}=24.2$ ) on the filter paper side and from 0.8 to 0.9 (luminosity $\mathrm{L}^{*}=42.7$ ) on the opposite side. In short, luminosity was confirmed to be largely different between the two sides of the collagen sheet with melanin added at $50 \%$. In contrast, little difference in luminosity (luminosity $\mathrm{L}^{*}=98.1$ on both sides) was found between the two sides of the collagen sheet with no melanin added (control). These data quantitatively showed that melanin was distributed more densely on the filter paper side of collagen sheets with melanin added.

In this study, the collagen sheets were used to study the effects of ultraviolet light on human skin of various types and age groups. Electron microscopy was performed with two collagen sheets prior to ultraviolet light irradiation: one was a collagen sheet with melanin added at $50 \%$ and the other was a collagen sheet Maillard cross-linked with a $10 \mathrm{mM}$ glyoxal solution. For comparison, the electron microscopy was performed on a collagen sheet that had no added melanin or cross-linkage (control). Fig. 2 shows the results of the electron microscopic observations of the surfaces and cross-sections of the collagen sheets.

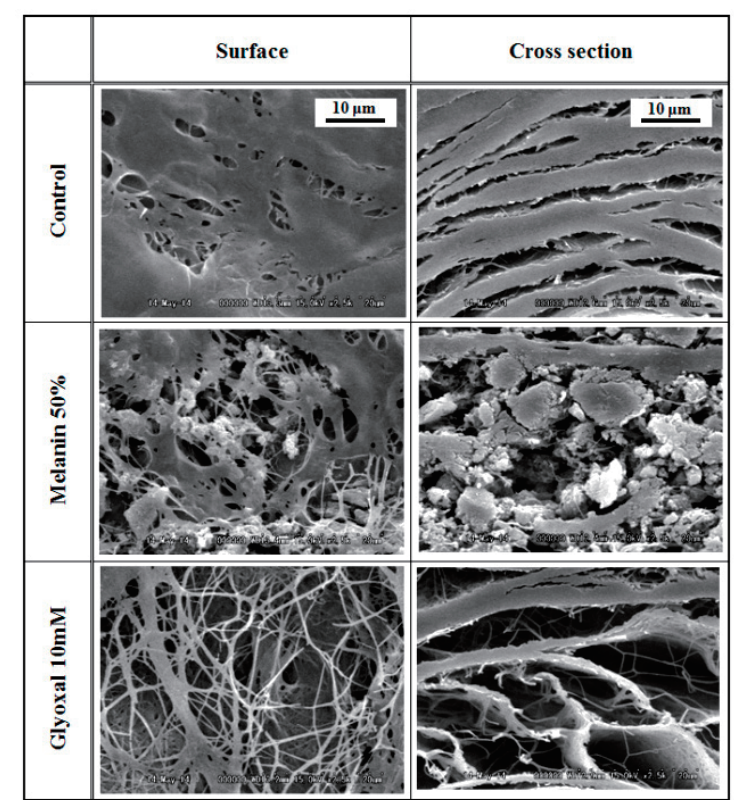

Fig. 2 Scanning electron micrographs (SEM) of the surface and cross-section of a collagen sheet before ultraviolet light irradiation $(\times 2,500)$.

An electron micrograph shows a relatively smooth surface of the collagen sheet that neither had added melanin nor was Maillard cross-linked (control). Furthermore, the cross-section of the collagen sheet showed a laminated structure with layers of two to four micrometers in thickness. In contrast, the collagen sheet with melanin added at $50 \%$ had 
many small lumps of two to six micrometers in diameter on the surface and in the cross-section. These lumps seemed to be aggregates of melanin. Also, the collagen sheet that was Maillard cross-linked with $10 \mathrm{mM}$ glyoxal showed fiber tissues that formed fine networks. These data revealed that Maillard cross-linking of collagen sheets affected sheet structure. The above results revealed that collagen sheets with melanin added or those that were Maillard cross-linked had structures that were largely different from that of the collagen sheet that had no added melanin and was not Maillard cross-linked.

\subsection{Changes in color hue of collagen sheets by ultra- violet light}

A study was conducted to determine how various levels of melanin and Maillard cross-linking influenced the color hue of collagen sheets after ultraviolet light irradiation. In the study, the duration of ultraviolet light irradiation was altered determine the effect on color hue. Fig. 3 shows how the reflectance spectrum changed with respect to the amount of melanin added to collagen sheets. Fig. 3 (a) shows the reflectance spectrum for collagen sheets before ultraviolet light irradiation, Fig. 3 (b) shows that of sheets irradiated for 25 hours, and Fig. 3 (c) shows that of sheets irradiated for 200 hours. The reflectance spectrum of a collagen sheet left shielded from light for 200 hours was also measured. The results are included in Fig. 3 (c).

The result revealed that reflectance substantially decreased for a wavelength range of 230-700 nm with increasing melanin amounts in collagen sheets before ultraviolet light irradiation [Fig. 3 (a)]. Moreover, the reflectance spectrum was flat without a peak for visible light $(380-780 \mathrm{~nm})$ in any sample. This means that the collagen sheets were achromatic (gray). In addition, the declining reflectance for the ultraviolet domain below $450 \mathrm{~nm}$ with increasing melanin levels revealed that melanin effectively absorbed ultraviolet light.

When ultraviolet light irradiation lasted for 25 hours [Fig. 3 (b)], reflectance decreased substantially from the levels observed before irradiation in the wavelength domain below $450 \mathrm{~nm}$, regardless of melanin amount. This means that collagen molecule chains were cross-linked by exposure to ultraviolet light, which allowed ultraviolet light to be absorbed more readily.

This tendency was more pronounced when irradiation lasted for 200 hours [Fig. 3 (c)]. In other words, long exposures to ultraviolet light irradiation further decreased reflectance for a wavelength range of 230-450 $\mathrm{nm}$. The collagen sheet that was shielded from light for 200 hours showed little difference in the reflectance spectrum compared with the collagen sheet that had no added melanin before irradiation. This revealed that ultraviolet light alone caused changes in the reflectance spectrum and heat had a minimal effect.
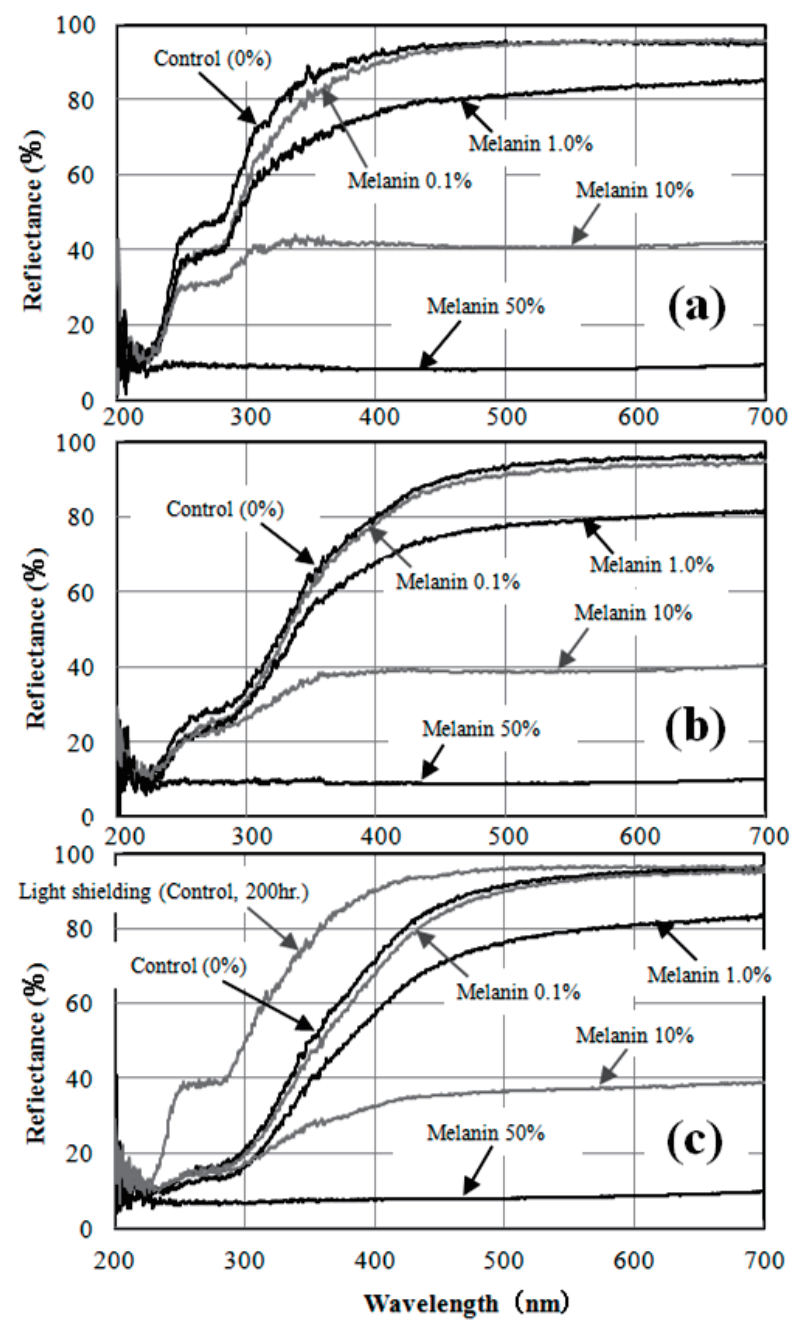

Fig. 3 Changes in the reflectance spectrum of melanin-added collagen sheets caused by ultraviolet light irradiation.

(a) Before irradiation, (b) irradiation for 25 hours, (c) irradiation for 200 hours

The reflectance spectra of Maillard cross-linked collagen sheets were also measured. Fig. 4 (a) shows the reflectance spectra for collagen sheets before ultraviolet light irradiation, Fig. 4 (b) shows those for collagen sheets irradiated for 25 hours, and Fig. 4 (c) shows those for collagen sheets irradiated for 200 hours. The results revealed that different glyoxal concentrations did not result in differences in the reflectance spectrum. In other words, changes in the level of Maillard cross-linking had relatively small effects on the reflectance spectrum. This is believed to result from glyoxal-induced glycosylation of some amino acids constituting collagen. The glycosylation made the reflectance spectrum less likely to change by ultraviolet light irradiation. Changes in the level of cross-linking resulted in smaller changes in reflectance spectrum than did changes in melanin level. In brief, changes in the level of Maillard cross-linking 
had little effect on color hue in collagen sheets.
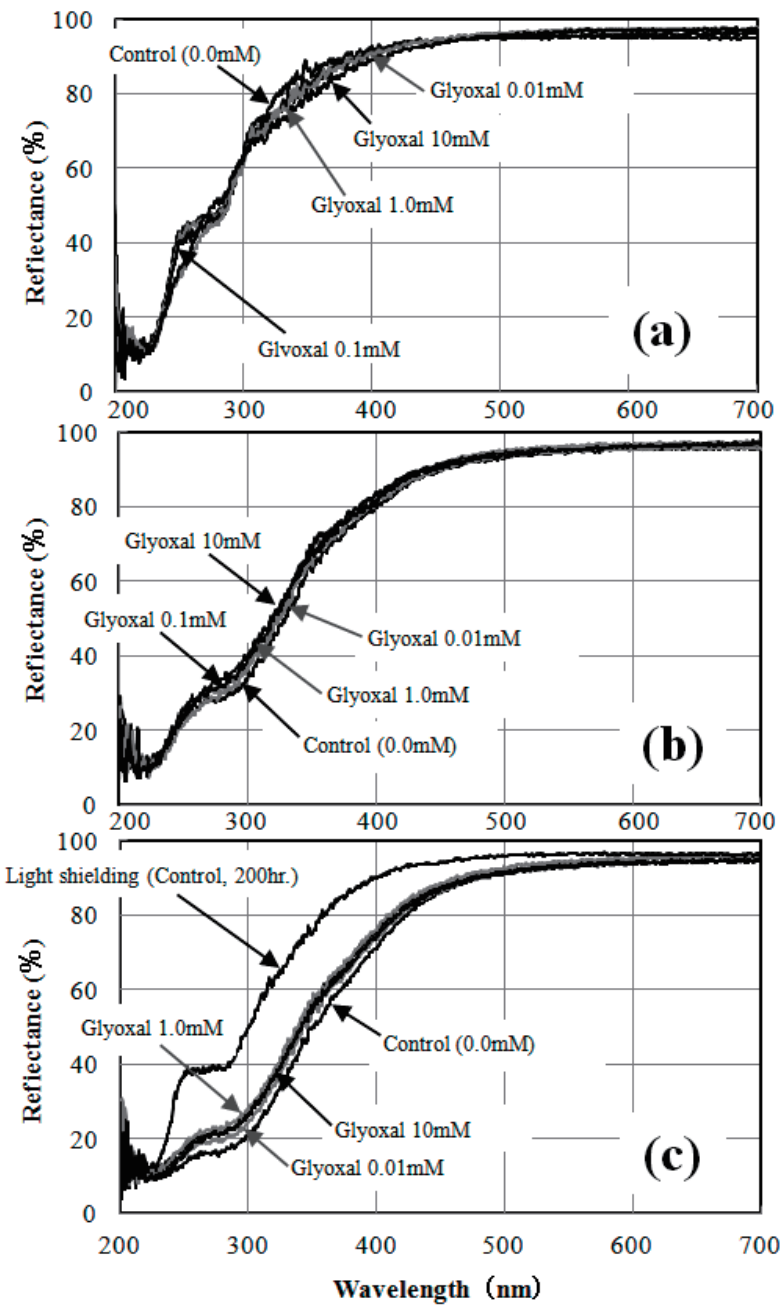

Fig. 4 Changes in the reflectance spectra of collagen sheets Maillard cross-linked with glyoxal caused by ultraviolet light irradiation.

(a) Before irradiation, (b) irradiation for 25 hours, (c) irradiation for 200 hours

However, long durations of ultraviolet light irradiation, such as 25 and 200 hours, of collagen sheets decreased reflectance for a wavelength domain below $450 \mathrm{~nm}$ compared with that of collagen sheets before irradiation. Similar to the results observed for melanin addition, a longer duration of ultraviolet light irradiation further decreased reflectance for a wavelength domain below $450 \mathrm{~nm}$ compared with collagen sheets before irradiation. This showed that exposure of collagen molecule chains to ultraviolet light altered their structure resulting in a better ability to absorb ultraviolet light This tendency was more pronounced when irradiation lasted for 200 hours. In short, reflectance was found to decrease further in a wavelength domain below $450 \mathrm{~nm}$. However, Maillard cross-linking had characteristically small effects, even when ultraviolet light irradiation lasted for 200 hours.

3.3 Effects of ultraviolet light on the structure of

\section{collagen molecule chains}

To study the effects of ultraviolet light on the structure of collagen molecule chains, collagen sheets were subjected to ultraviolet light irradiation for up to 200 hours. Collagen sheets after ultraviolet light irradiation were extracted with acetic acid and the extracts were analyzed by biuret and ninhydrin reactions.

Fig. 5 (a) shows the results of the biuret analysis for extracts derived from melanin-added collagen sheets after ultraviolet light irradiation. The results showed that the protein concentration decreased in the extracts from all collagen sheets, except that the extract from the collagen sheet with melanin added at $50 \%$, as the duration of ultraviolet light irradiation increased up to 25 hours. The protein concentration then increased in the extracts when ultraviolet light irradiation continued for up to 200 hours. In contrast, the protein concentration decreased in the extract from the collagen sheet with melanin added at the highest level of $50 \%$ as ultraviolet light irradiation increased in duration, even for up to 200 hours.
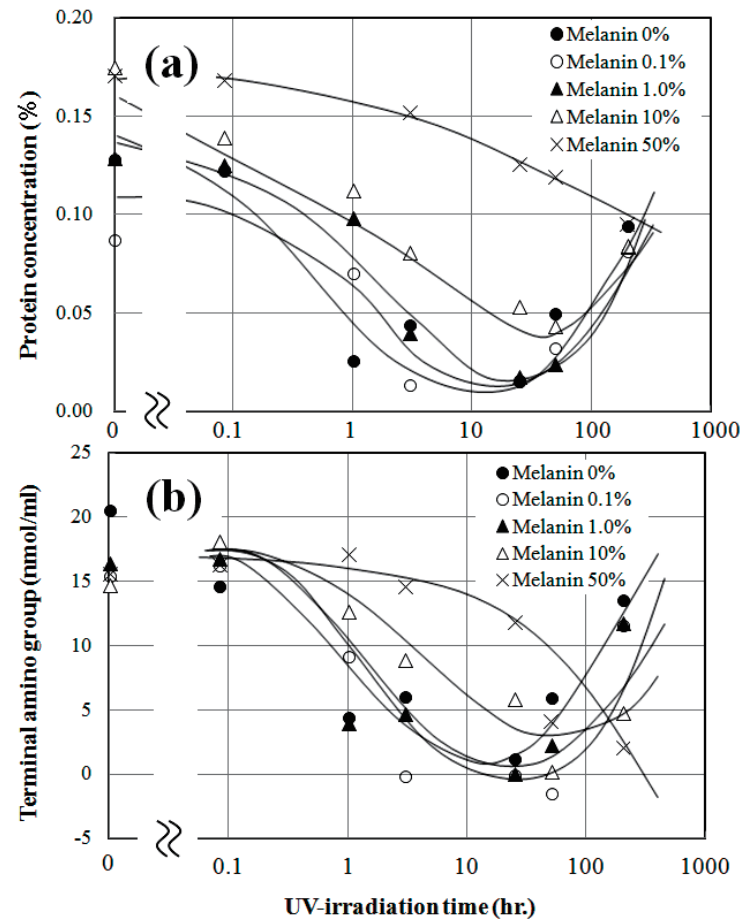

Fig. 5 Changes in artificial collagen skins with melanin pigment added at various levels for various durations of ultraviolet light irradiation.

(a) Protein concentration, (b) terminal amino acid concentration

The protein concentration increased with increasing amounts of melanin for ultraviolet light irradiation lasting up to 25 hours. This showed that the melanin effectively absorbed ultraviolet light to arrest cross-linking in collagen molecule chains by ultraviolet light. However, ultraviolet light irradiation for 200 hours enhanced the degradation of 
collagen molecule chains in collagen sheets with smaller amounts of melanin added, resulting in increased protein concentration.

As described above, ultraviolet light irradiation lasting up to 25 hours mainly cross-linked collagen molecule chains in collagen sheets with melanin added at $10 \%$ or lower, and longer durations of ultraviolet light irradiation mainly cleaved collagen molecule chains. In contrast, the collagen sheet with melanin added at $50 \%$ was not susceptible to ultraviolet light and collagen molecule chains in the sheet were not readily cleaved even when ultraviolet light irradiation continued for 200 hours.

The terminal amino acid concentrations of the extracts were also analyzed by the ninhydrin reaction, and the results are shown in Fig. 5 (b). The terminal amino acid concentration of the extracts showed a similar tendency to that of the protein concentration. In short, melanin addition was highly effective in suppressing damage to collagen molecule chains by ultraviolet light.

Maillard cross-linked collagen sheets were also analyzed after ultraviolet light irradiation. The protein and terminal amino acid concentrations of acetic acid extracts from the treated collagen sheets were examined. Fig. 6 (a) shows the relationship between protein concentration in the extracts from the Maillard cross-linked collagen sheets and the duration of ultraviolet light irradiation. The protein concentration in the extracts decreased with increasing glyoxal concentrations, and was relatively low when the glyoxal concentration was as high as 1 or $10 \mathrm{mM}$. In other words, Maillard cross-linking by glyoxal made collagen sheets less readily extractable with acetic acid. However, even when the glyoxal concentration was 1 or $10 \mathrm{mM}$, ultraviolet light irradiation for as long as 200 hours tended to degrade collagen molecule chains and increase the protein concentration.

The protein concentration decreased in extracts from collagen sheets treated with a glyoxal solution of $0.1 \mathrm{mM}$ or lower as ultraviolet light irradiation increased to 25 hours in duration. Furthermore, the protein concentration increased when ultraviolet light irradiation continued for longer than 25 hours. In other words, ultraviolet light irradiation for up to 25 hours cross-linked collagen molecule chains in collagen sheets Maillard cross-linked at low levels, and ultraviolet light irradiation for longer than 25 hours readily degraded collagen molecule chains.

The terminal amino acid concentrations of collagen molecule chains of extracts were then analyzed by the ninhydrin reaction and the results are shown in Fig. 6 (b). The results were consistent with those observed for the protein concentration described above. In brief, collagen sheets treated with glyoxal solutions of $1 \mathrm{mM}$ or higher were difficult to extract with acetic acid and not susceptible to ultraviolet light. However, ultraviolet light irradiation for 200 hours was found to cleave collagen molecule chains.

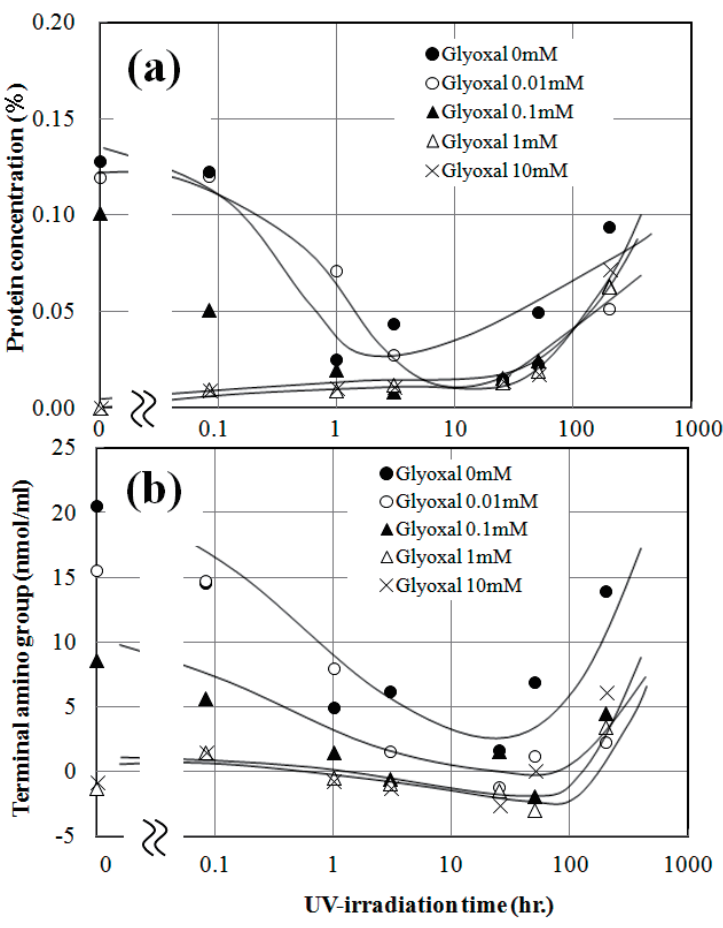

Fig. 6 Changes in artificial collagen skins cross-linked with glyoxal at various levels for various durations of ultraviolet light irradiation.

(a) Protein concentration, (b) terminal amino acid concentration

The data presented above quantitatively showed that ultraviolet light irradiation initially cross-linked collagen molecule chains to form larger molecules and gradually degraded them to smaller molecules. Moreover, the collagen sheets with more melanin added or those that were Maillard cross-linked at higher levels were less affected by ultraviolet light.

\subsection{Electrophoretic analysis of damage to collagen molecule chains by ultraviolet light}

Electrophoretic analysis was performed to study damage to collagen molecule chains caused by ultraviolet light in finer detail. Fig. 7 shows the electrophoretic band patterns for extracts from melanin-added collagen sheets after ultraviolet light irradiation. From left to right, the results are shown for collagen sheets before irradiation (a), for those irradiated for 25 hours (b), and for those irradiated for 200 hours (c). All samples showed many bands for collagen molecule chains before ultraviolet light irradiation. The level of melanin addition had no effect on the appearance of bands.

After ultraviolet light irradiation for 25 hours, bands for collagen molecule chains and their degradation products were not observed for collagen sheets with melanin added at $0 \%$, $0.1 \%$, and $1.0 \%$. In contrast, a number of bands for collagen molecule chains were observed for collagen sheets with more 

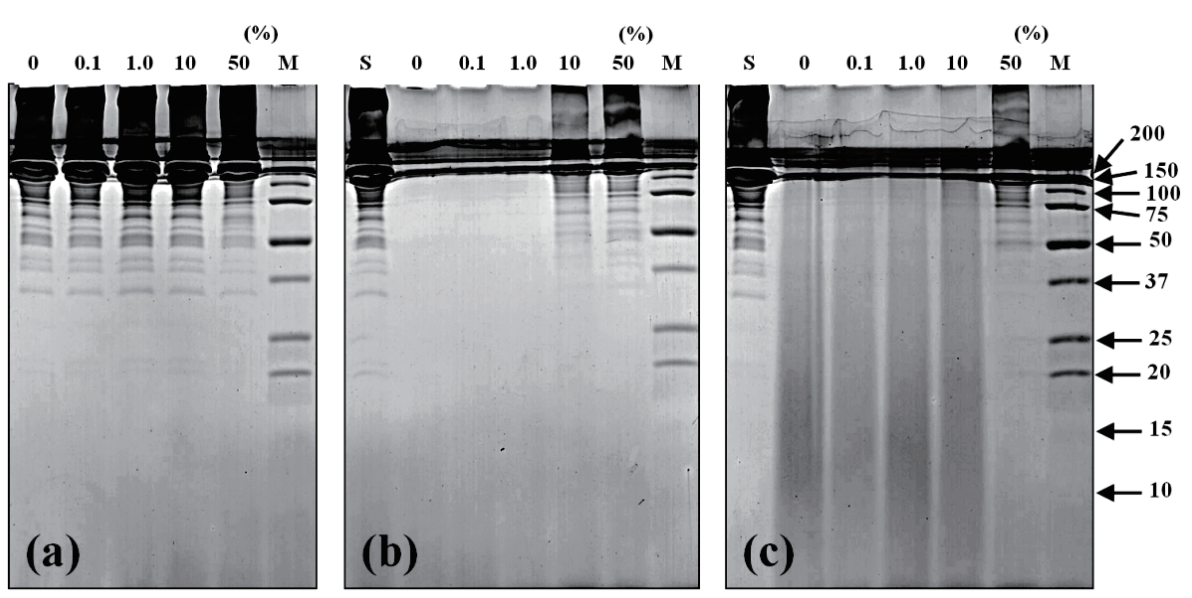

Fig. 7 SDS-PAGE for melanin-added collagen sheets after ultraviolet light irradiation:

(a) 0 hours, (b) 25 hours, (c) 200 hours; S: Light shielded sample, M: molecular marker

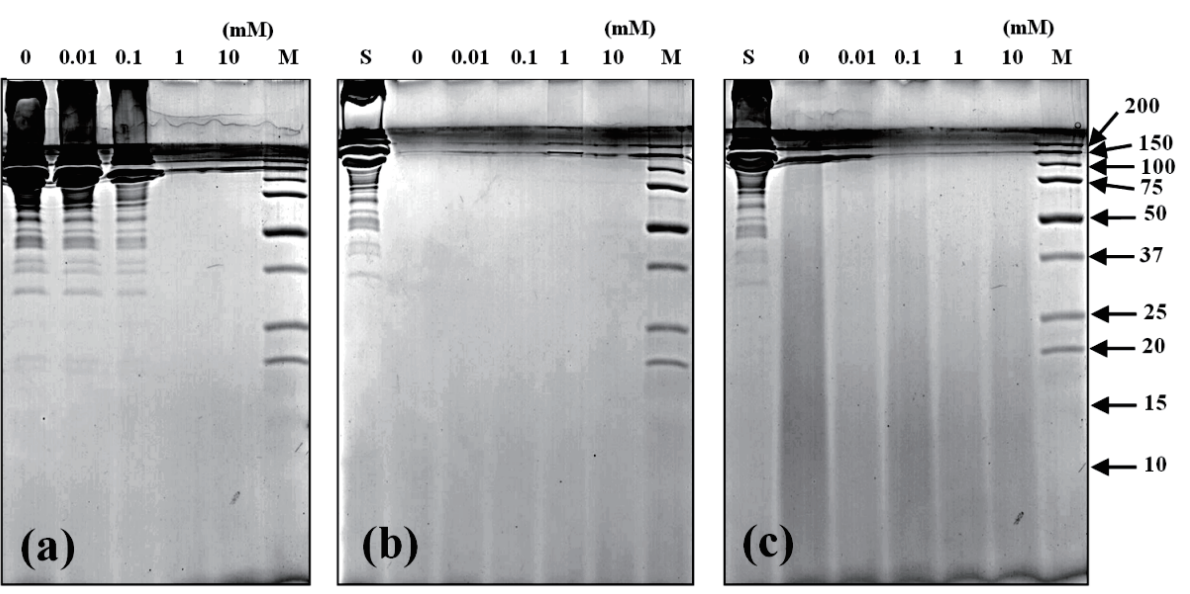

Fig. 8 SDS-PAGE for Maillard cross-linked collagen sheets with glyoxal after ultraviolet light irradiation:

(a) 0 hours, (b) 25 hours, (c) 200 hours; S: Light shielded sample, M: molecular marker

melanin added at $10 \%$ and $50 \%$.

After 200 hours of ultraviolet light irradiation, degradation products from collagen molecule chains ranging from 10-20 KDa were observed, except for the collagen sheet with melanin added at $50 \%$. No band for collagen molecule chains was observed for the collagen sheets. In contrast, the collagen sheet with melanin added at 50\% showed bands for collagen molecule chains even after ultraviolet light irradiation for 200 hours.

As described above, damage to collagen molecule chains largely varied by the duration of ultraviolet light irradiation. In general, ultraviolet light irradiation for 25 hours mainly formed cross-linking, which made extraction difficult, and irradiation for 200 hours characteristically yielded many degradation products. Furthermore, collagen sheets with more melanin added tended to be less susceptible to ultraviolet light and the collagen sheet with melanin added at 50\% was minimally affected by ultraviolet light.

Fig. 8 shows the results of electrophoresis for collagen sheets Maillard cross-linked with glyoxal. From left to right, the results are shown for collagen sheets before irradiation (a), for those irradiated for 25 hours (b), and for those irradiated for 200 hours (c). Bands of collagen molecule chains were observed for collagen sheets before ultraviolet light irradiation that were treated with relatively low concentrations, i.e., $0-0.1 \mathrm{mM}$, of glyoxal solutions. However, no band of collagen molecule chains was observed for collagen sheets treated with glyoxal solutions of 1 or $10 \mathrm{mM}$. In short, Maillard cross-linking formed by glyoxal made collagen difficult to extract with acetic acid.

No band of collagen molecule chains or their degradation products was observed for collagen sheets after 25 hours of ultraviolet light irradiation regardless of glyoxal concentration. This means that cross-linking formed by ultraviolet light in addition to Maillard cross-linking made collagen difficult to extract with acetic acid. In other words, extracts from collagen sheets were more difficult to obtain with acetic acid by cross-linking formed by ultraviolet light even when a glyoxal solution of 0 or $0.01 \mathrm{mM}$ was used. Furthermore, after 200 hours of ultraviolet light irradiation, degradation products from collagen molecule chains appeared and no band was found for collagen molecule chains.

As described above, extracts from collagen sheets Maillard cross-linked with glyoxal were difficult to obtain 
with acetic acid. Except collagen sheets treated with a glyoxal solution of 0.01 or $0.1 \mathrm{mM}$, bands for collagen molecule chains were not clearly observable. When ultraviolet light irradiation was further extended in duration, no band was observed for any samples. However, ultraviolet light irradiation for 200 hours yielded degradation products from collagen molecule chains.

As described above, collagen sheets Maillard crosslinked at higher levels to reflect aging were less susceptible to ultraviolet light. However, the human skin of an actual living body is believed to become more susceptible to ultraviolet light with advancing ages. In short, older individuals are more easily damaged by ultraviolet light resulting in wrinkles, sagging, and blemishes. However, the evaluation of collagen sheet damage by ultraviolet light in this study revealed that collagen sheets Maillard cross-linked at higher levels were less susceptible to ultraviolet light.

The reason for the difference may be the absence of fibroblasts in collagen sheets, in contrast to the presence of not only collagen but also fibroblasts in the skin of a living human. Fibroblasts secrete degradation enzymes, such as collagenase and elastase, in the skin. These enzymes recognize cross-linked products formed via cross-linking in collagen and elastin as xenobiotics. Fibroblasts are believed to secrete more degradation enzymes as the level of cross-linking increases in the skin. Moreover, the Maillard reaction in collagen fibers is known to arrest the scaffold function of fibroblasts ${ }^{11)}$.

In this study, there was no secretion of collagenase or elastase because the collagen sheets lacked fibroblasts. This difference may explain the differences between the behavior of collagen sheets and that of human skin.

\section{Conclusions}

Collagen sheets with melanin added or those that were Maillard cross-linked with glyoxal were prepared to simulate various skin types or ages. The treated collagen sheets were used to examine human skin damage caused by ultraviolet light.

(1) The collagen sheet cross-section morphology showed a laminated structure with layers of 2-4 micrometers in thickness. Collagen sheets with melanin added showed many lumps of 2-6 micrometers in diameter, which seemed to be melanin aggregates. Maillard cross-linked collagen sheets had a network structure of fibers $0.6-0.8$ micrometers in diameter.

(2) Extracts from collagen sheets before ultraviolet light irradiation were analyzed. Ultraviolet light irradiation for up to 25 hours mainly resulted in cross-linking in collagen sheets with melanin added at $10 \%$ or lower, and irradiation for 200 hours mainly resulted in cleaved collagen molecule chains.

(3) Ultraviolet light irradiation in general exerted relatively little effect on the molecular structure of the collagen sheet with melanin added at 50\%. Ultraviolet light did not cleave collagen molecule chains, although it enhanced cross-linking in collagen molecule chains.

(4) Extractions from collagen sheets Maillard cross-linked with glyoxal solutions of $0.1 \mathrm{mM}$ or higher were difficult to obtain with acetic acid. However, ultraviolet light irradiation for 200 hours was found to cleave collagen molecule chains.

\section{References}

1) M. H. Repacholi, "Global solar UV index." Radiation Protection Dosimetry, Vol. 91, pp. 307-311 (2000).

2) P. Koepke, A. Bais, H. De Backer, X. De Cabo, P. Eckert, P. Eriksen, D. Gillotay, A. Heikkilae, and M. Weber, "Comparison of Models Used for UV Index Calculations", Photochemistry and Photobiology, Vol. 67, pp. 657-662 (1998).

3) A. Kawada, "Protection and Therapy of Photoaging", Anti Aging Medicine, Vol. 8, pp. 88-91 (2011).

4) K. Motoyoshi, Y. Ota, Y. Takuma, and M. Takenouchi, "Wrinkles from UVA Exposure", Cosmetics and Toiletries, Vol. 113, pp. 51-56,58 (1998).

5) A. V. Rawlings, "Ethnic Skin Types: Are there Differences in Skin Structure and Function?", IFSCC Magazine, Vol. 9, pp. 3-13 (2006).

6) R. Pugashetti, H. I. Maibach, "Variations in Pigmentation and Ultrastructural Skin Differences Among Ethnic Groups", Cosmetics and Toiletries, Vol. 125, pp. 18,20-23 (2010).

7) P. Alexander, "Ageing skin", Causes \& effects, Manufacturing Chemist, Vol. 56, pp. 33,35 (1985).

8) T. Takahashi, T. Kondo, K. Tanaka, S. Hattori, S. Irie, S. Kudoh, S. Imura, and H. Kanda: "Measurement of solar UV radiation in Antarctica with collagen sheets", Photochemical \& Photobiological Sciences, Vol. 11, pp. 1193-1200 (2012).

9) T. Takahashi, T. Kondo, K. Tanaka, S. Hattori, S. Irie, S. Kudoh, S. Imura, and H. Kanda: "Using collagen artificial skin to estimate the protection effects of UV-cut materials against sunlight under the Antarctic ozone hole", Polymer Degradation and Stability, Vol. 97, No. 6, pp. 1002-1009 (2012).

10) U. K. Laemmli, "Cleavage of Structural Proteins during the Assembly of the Head of Bacteriophage T4", Nature, Vol. 227, pp. 680-685 (1970).

11) E. Kawano, S. Takahashi, Y. Sakano and D. Fujimoto, "Nonenzymatic glycation alters properties of collagen as a substratum for cells", Matrix, Vol. 10, pp. 300-305 (1990). 